\title{
Nanotechnologie - eine Herausforderung für die Arbeitsmedizin
}

\author{
Nanotechnology - New Challenges in Occupational Medicine
}

Autoren

Institut
M. Nasterlack, C. Oberlinner, B. van Ravenzwaay, R. Landsiedel, S. Lang

Abteilung Arbeitsmedizin und Gesundheitsschutz, BASF SE, Ludwigshafen
Bibliografie

DOI http://dx.doi.org/

$10.1055 / \mathrm{s}-0030-1256105$

Akt Dermatol 2011; 37:

81-86 @ Georg Thieme

Verlag KG Stuttgart · New York

ISSN 0340-2541

\section{Korrespondenzadresse}

PD Dr. med.

Christoph Oberlinner

Abteilung Arbeitsmedizin

und Gesundheitsschutz

BASF SE

GUA/B - H306

67056 Ludwigshafen

christoph.oberlinner@basf.com

\section{Zusammenfassung \\ $\nabla$}

Als Nanomaterialien werden solide partikuläre Materialien bezeichnet, die nanoskalig - also mit mindestens einer Dimension kleiner als $100 \mathrm{~nm}$ $\left(100 \times 10^{-9} \mathrm{~m}\right)$ - hergestellt wurden (ICCA-Definition). Nanoskaliges Material natürlichen Ursprungs wird üblicherweise nicht als Nanomaterial, sondern als ultrafeines Material (Ultrafeinstaub) bezeichnet. Nanomaterialien können chemische und physikalische Eigenschaften haben, die sich von denen der gleichen Materialien in nichtnanoskaliger Größe unterscheiden. Diese geänderten oder neuen Materialeigenschaften werden in der Nanotechnologie gezielt genutzt. Neben den gewünschten Materialeigenschaften können Nanomaterialien auch toxikologische Eigenschaften aufweisen, die aus den Eigenschaften des nichtnanoskaligen Materials gleicher $\mathrm{Zu}$ -

\section{Einleitung}

\section{$\nabla$}

Die Begriffe „Nanopartikel“, „Nano-Objekte“ oder „Nanomaterialien“ sind international noch nicht abschließend definiert. Nach einer ISOVornorm werden hierunter solide partikuläre Materialien verstanden, die in mindestens einer Dimension eine Größe zwischen 1 und $100 \mathrm{~nm}$ $\left(1 \mathrm{~nm}=10^{-9} \mathrm{~m}\right)$ aufweisen. In der chemischen Industrie werden Nanomaterialien als solide, partikuläre Substanzen definiert, die absichtlich nanoskalig (intentionally manufactured) hergestellt wurden; Zusammenlagerungen von Nanomaterialien (Aggregate und Agglomerate) werden ebenfalls als Nanomaterialien definiert (ICCA 2010).

Nanoskalige Materialien weisen zum Teil völlig andere Eigenschaften auf als die gleiche chemische Substanz in größerer Dimension oder in gelöster Form. Dieser Umstand, der Nanomaterialien zum einen technisch und wissenschaftlich so sammensetzung nicht unmittelbar abzuleiten sind. Aus In-vitro- und Tierversuchen ergibt sich der Verdacht, dass manche Nanomaterialien, wie manche gröbere Partikel auch, entzündungsfördernd und fibrogen sein können; für Nanomaterialien werden aber eine stärkere Wirkung und andere Wirkorte im Körper diskutiert. Die Beurteilung von Expositionen gegenüber Nanomaterialien gestaltet sich schwierig. Bis heute existieren keine Messverfahren, mit denen Anzahl, Oberfläche oder auch chemische Identität nach einem standardisierten Verfahren bestimmt werden könnten. Da bislang zu Gesundheitswirkungen von Nanomaterialien keine Erfahrungen am Menschen vorliegen, ist eine gezielte arbeitsmedizinische Vorsorge zur Früherkennung spezifischer Effekte nicht möglich. Somit kommt beim beruflichen Umgang mit Nanomaterialien dem Arbeitsschutz höchste Bedeutung zu.

faszinierend macht, begründet zum anderen die Besorgnis, dass sie auch bislang unbekannte oder unbeachtete toxikologische Eigenschaften aufweisen können [1,2].

Die Nanotechnologie gilt als die Zukunftstechnologie, die Grundlagen für immer kleinere Datenspeicher mit immer größerer Speicherkapazität, für hochwirksame Filter zur Abwasseraufbereitung, für fotovoltaische Fenster, für Werkstoffe, aus denen sich in der Automobilindustrie ultraleichte Motoren und Karosserieteile oder Lacke mit erhöhter Kratz und Abriebfestigkeit fertigen lassen. Auch bei künstlichen Gelenken, die durch organische nanostrukturierte Oberflächen für den menschlichen Körper verträglicher sind, bestehen praktische Anwendungen [3]. Gleichzeitig stellt der Umgang mit Nanomaterialien in der Arbeits- und Umwelt unter Umständen auch eine besondere - wenngleich nicht immer neue - arbeitsmedizinische Herausforderung dar [4]. 


\section{Vorkommen von Nanomaterialien} $\nabla$

Menschen sind schon zu allen Zeiten und überall gegenüber nanoskaligen Materialien exponiert gewesen. Diese ultrafeinen Stäube stammen beispielsweise aus natürlichen oder anthropogenen Verbrennungsprozessen, Vulkanausbrüchen etc. Typische exponierte Berufstätigkeiten sind z.B. Schweißen, Löten und Brennschneiden, aber auch beim Mahlen von Getreide, Pigmenten und anderen Produkten können Stäube entstehen, deren Größenverteilung bis hinunter in die Nano-Dimension reicht. Die weitaus höchste nichtberuflich bedingte Exposition gegenüber ultrafeinen Stäuben wird beim Tabakrauchen erreicht.

Von diesen natürlichen oder unbeabsichtigt produzierten Partikeln sind die gezielt hergestellten Nanomaterialien („engineered nanonanomaterials“) zu unterscheiden. Nanomaterialien können als einfache kugelige Kohlenstoffpartikel vorliegen (carbon black). Kohlenstoff kann aber auch in Röhrenform („Carbon-Nanotubes“) konfiguriert sein und dabei eine oder mehrere Wandschichten aufweisen. Deren Eigenschaften können durch gezielte Modifikationen bestimmt werden, um beispielsweise die räumliche Konfiguration, die elektrische Leitfähigkeit oder katalytische Eigenschaften zu modifizieren. Nicht auf Kohlenstoffbasis, sondern typischerweise aus Halbleitermaterialien werden sogenannte Quantenpunkte („quantum dots“) hergestellt. Hierbei handelt es sich um nur aus ca. 100-100000 Atomen bestehende Strukturen mit einem Durchmesser von 10 bis $50 \mathrm{~nm}$.

\section{Anwendung der Nanomaterialien \\ $\nabla$}

Es existieren keine genauen Angaben, wie viele verschiedene Nanomaterialien auf dem Markt vorhanden sind und in welchen Anwendungen sie genau vorkommen. In verbrauchernahen Produkten aus kohlefaserverstärkten Kunststoffen wie Tennis- und Golfschläger oder Skiern werden beispielsweise Kohlenstoffröhrchen, sogenannte Nanotubes, zur Erhöhung der Festigkeit eingesetzt. Nanosilber wird als Biozid zur Verhinderung des Schweißgeruchs in Textilien eingesetzt.

Nanoskalige $\mathrm{TiO}_{2}$ - und ZnO-Pigmente werden zur Erzielung hoher Sonnenschutzfaktoren ohne die unerwünschten Farbeindrücke von diversen Ölen in Sonnenschutzmitteln eingesetzt.

\section{Beurteilung der Exposition \\ $\nabla$}

Bis heute existiert kein Messverfahren, mit dem Partikelzahl, Oberfläche und chemische Identität standardisiert und in einem einzigen Messvorgang bestimmt werden könnten. Eine besondere Herausforderung in der Routine besteht bei Arbeitsplatzmessungen auch darin, Nanomaterial von dem im Hintergrund immer vorhandenen ultrafeinen Staub zu unterscheiden.

Folgende Expositionsmaße und -charakteristika sind sinnvoll bzw. wünschenswert: (1) Partikelmasse, (2) Partikelzahl, (3) Partikel-Gesamtoberfläche, (4) Partikelgeometrie, (5) Partikelchemie und (6) eventuelle Kontaminationen (z.B. aus Katalysatoren) [5].

Die Partikelmasse ist grundsätzlich nach üblichen gravimetrischen Verfahren bestimmbar. Sie stellt allein aber keine ausreichende Expositionsbeschreibung dar, da der nanoskalige Anteil in einer gegebenen Größenverteilung zwar ggf. die Hauptzahl der Partikel, aber nur einen Bruchteil ihrer Gesamtmasse ausmacht.
Für die Messung der Partikelzahl stehen mehrere unterschiedliche Verfahren zur Verfügung, deren Ergebnisse untereinander jedoch nur mit großen Einschränkungen vergleichbar sind. Komplizierend kommt hinzu, dass die Messmethoden selbst durch ihren Energieeintrag das Größenspektrum des Messgutes verändern können.

Über die Partikelgeometrie kann derzeit nur mit elektronenmikroskopischen Verfahren eine Aussage getroffen werden. Somit ist auch die Berechnung von Partikeloberflächen kein triviales Unterfangen.

Insgesamt liegen nur spärliche vergleichende Messdaten sowohl aus der allgemeinen Umwelt als auch von relevanten Arbeitsplätzen vor. Messungen im Freien fernab von Verkehrsstraßen und in Innenräumen ohne besondere Belastungen haben Partikelzahlen bis ca. $50000 / \mathrm{cm}^{3}$ erbracht. In industrieller Umgebung wurden ohne besondere Aktivitäten bis zu $450000 / \mathrm{cm}^{3}$ gemessen, während Abfüllarbeiten von ultrafeinem Titandioxid stiegen die Werte bis $460000 / \mathrm{cm}^{3}$. In Backstuben traten Belastungen bis $640000 / \mathrm{cm}^{3}$ auf und bei Schweißerarbeiten sowie in der Ausatemluft eines Rauchers überstieg die gemessene Partikelzahl $107 / \mathrm{cm}^{3}$.

\section{Biomonitoring}

Methoden zur Messung der inneren Exposition mit Nanomaterialien („Biomonitoring“) am Menschen sind derzeit nicht evaluiert. Verfahren wie radioaktive Markierung sind aus naheliegenden Gründen nicht einsetzbar und die Konzentrationsbestimmung der chemischen Grundbestandteile solcher Partikel in Blut, Urin oder anderen Körpermaterialien ist angesichts des winzigen Massebeitrags, den selbst eine vergleichsweise hohe Partikelzahl im Nanobereich darstellen würde, in den meisten Fällen nicht zielführend. Eine Ausnahme stellt gegebenenfalls eine exzessiv hohe Exposition dar. Dies wurde in einer Kasuistik beschrieben, wo es nach Aufsprühen von frisch in einem Lichtbogenprozess erzeugten Nickel-Nanomaterialien auf Metalloberflächen zu einer schweren akuten Intoxikation mit Todesfolge kam [6]. Die retrospektiv im Urin gemessene Nickelbelastung lag allerdings in einer Größenordnung, wie sie auch bei tödlichen Nickelvergiftungen aus anderen Quellen gefunden worden waren. Ansonsten kann Biomonitoring versucht werden, wenn es sich bei dem Nanomaterial um eine chemische Substanz handelt, die üblicherweise im menschlichen Körper nicht oder fast nicht nachweisbar ist und für die eine hinreichend sensitive Analysenmethode besteht.

\section{Potenzielle Gesundheitseffekte von Nanomaterialien $\nabla$}

Wie bereits beschrieben können sich die Eigenschaften von Nanomaterialien aufgrund ihrer geringen Größe im Vergleich zu herkömmlichen Materialien gleicher chemischer Zusammensetzung unterscheiden. Es hat sich jedoch auch ebenso gezeigt, dass Nanomaterialien hinsichtlich ihres toxischen Potenzials keine einheitliche Gruppe darstellen, sondern einzelne Nanomaterialien sehr unterschiedliches Verhalten und unterschiedliche Wirkungen im Körper zeigen können [7]. Es gibt daher keine „nanotoxikologische“ Wirkung per se, sondern die Wirkung einzelner Nanomaterialien, die sich voneinander unterscheiden. Gleichwohl ist es möglich, für bestimmte Wirkungen Aussagen 


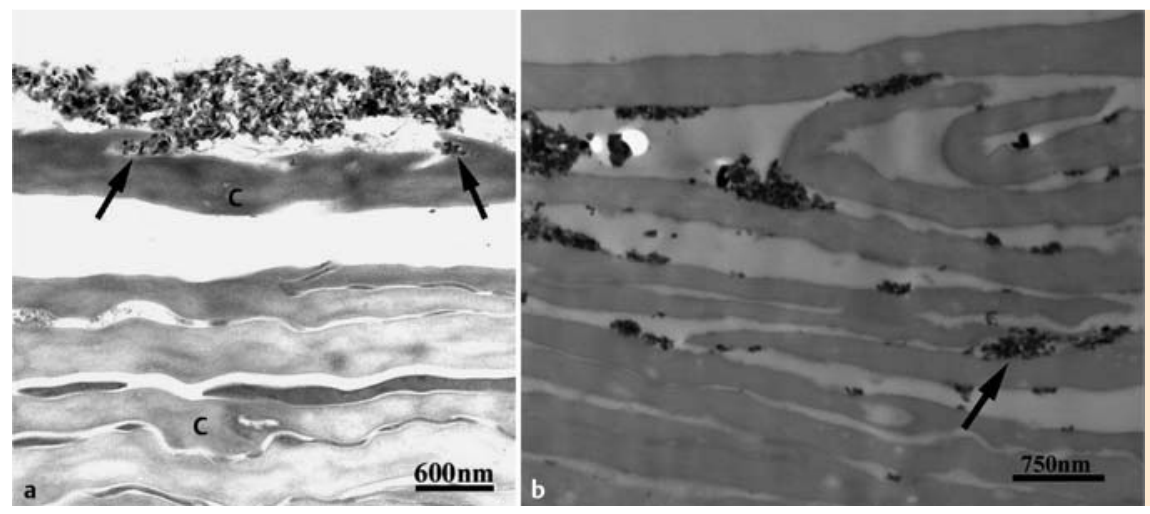

Abb. 1 Transmissionselektronenmikroskopie (TEM)-Bild von Schweinehaut 48 Stunden nach Applikation einer Sonnencreme mit Titandioxidnanopartikeln. a Haut ohne UVB-Bestrahlung; das Titandioxid befindet sich auf der Hautoberfläche. b UVB-bestrahlte Haut; das Titandioxid wurde in der Tiefe von bis zu 6-8 Zellschichten gefunden.
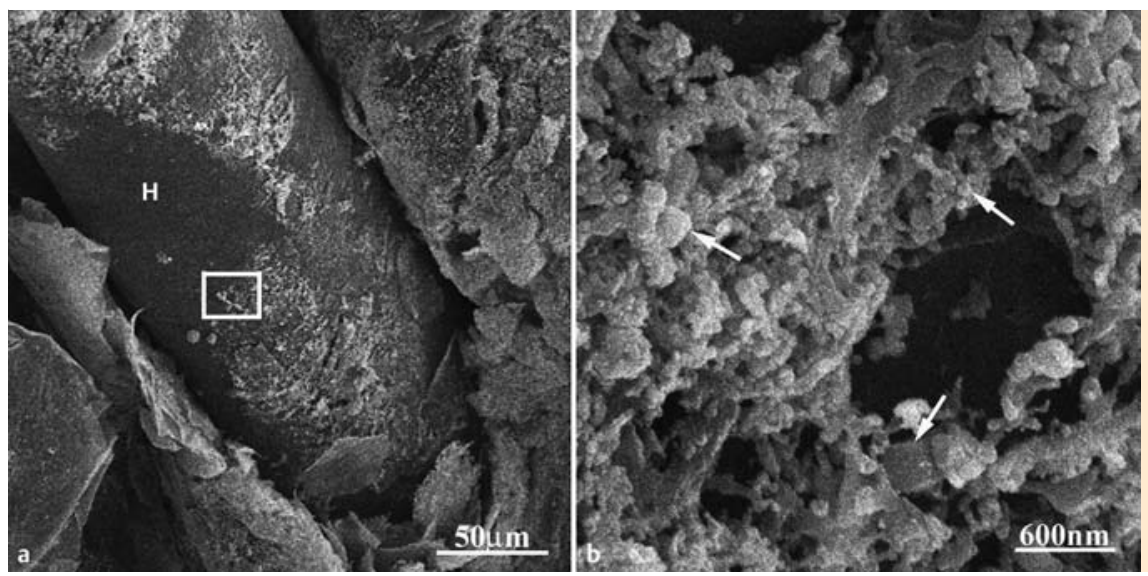

Abb. 2 TEM-Bild von Schweinehaut 48 Stunden nach Applikation einer Sonnencreme mit Zinkoxidnanopartikeln. a Zinkoxid auf dem Haarschaft. b Ausschnittsvergrößerung.

über Gruppen von Nanomaterialien - nicht aber für die Gruppe aller Nanomaterialien - zu machen.

Unter den möglichen Aufnahmewegen wurde insbesondere die Frage der Hautgängigkeit intensiv untersucht:

Nach den bisher vorliegenden Ergebnissen kann hierzu festgestellt werden, dass Nanomaterialien im Allgemeinen nicht hautgängig sind. Nur für einige spezielle, insbesondere sehr kleine, beschichtete Nanomaterialien konnte eine Aufnahme durch die Haut gezeigt werden $[8,9]$; die Mehrzahl der getesteten Nanomaterialien zeigte hingegen nur eine Penetration in - aber nicht durch - die oberen toten Hautschichten [10]. Für mehrere Nanomaterialien wurde die Ansammlung in den Haarfollikeln der Haut beschrieben [11].

Die Hautgängigkeit von $\mathrm{TiO}_{2}$ - und $\mathrm{ZnO}-$ Nanomaterialien, die in Sonnenschutzmitteln verwendet werden, wurde eingehend untersucht. Bereits 2006 berichteten Gamer et al. [12], dass diese nicht durch intakte Schweinehaut passieren. Abb. 1 und - Abb. 2 zeigen Schweinehaut 48 h nach Applikation einer Sonnencreme mit $\mathrm{TiO}_{2}$ - bzw. ZnO-Nanopartikeln. Bei UVB-bestrahlter Haut wurde das Titanoxid lediglich bis zu einer Tiefe von bis zu 6-8 Zellschichten gefunden.

Cross et al. [13] zeigten 2007, dass ZnO aus der O/W-Emulsion einer Sonnencreme zwar in die toten Schichten, nicht jedoch durch Menschenhaut penetrieren. Neuere Untersuchungen von $\mathrm{TiO}_{2}$ - und $\mathrm{ZnO}$-Nanomaterialien in $\mathrm{O} / \mathrm{W}$ - und $\mathrm{W} / \mathrm{O}$-Formulierungen bestätigten das Eindringen in die oberen Schichten intakter Schweinehaut. In sonnenverbrannter Schweinehaut drangen die Nanomaterialien tiefer ein, es wurde jedoch auch hier kein Durchtritt in das lebende Gewebe beobachtet $[14,15]$.

Zurzeit wird noch die Hautgängigkeit unter speziellen Bedingungen wie mehrtätiger Exposition und mechanisch beanspruchter Haut untersucht [16]. Überwiegend wird jedoch die systemische
Exposition gegenüber Nanomaterialien durch dermale Aufnahme als gering angesehen.

Aus arbeitsmedizinischer Sicht ist vor allem der inhalative Aufnahmeweg von Bedeutung. Der Übertritt einiger Nanomaterialien in die Blutbahn und die Verteilung in andere Organsysteme einschließlich des Bulbus olfactorius als vorgestülptem Teil des Gehirns wurde im Tierversuch nach inhalativer Exposition demonstriert, wobei die pathophysiologische Relevanz dieses Vorgangs ebenso ungeklärt ist wie die Frage, inwieweit diese Organverteilung auch für ultrafeines Material aus natürlichen Quellen stattfindet.

In zahlreichen In-vitro-Versuchen wurden Effekte auf die Zellvitalität, Phagozytoseaktivität, Bildung von Botenstoffen und Markern für oxidativen Stress untersucht und - je nach eingesetztem System und Partikeltyp - mehr oder weniger ausgeprägt gefunden. Allerdings sind aus diesen Versuchen in der Regel keine validen Schlussfolgerungen auf zu erwartende Gesundheitseffekte im intakten Organismus abzuleiten [17]. Solche Methoden können erst dann als Screening-Methoden eingesetzt werden, wenn die Relevanz der in vitro beobachteten Effekte und die Kinetik der Nanomaterialien im Körper bekannt ist. Um eine größere Zahl verschiedener Materialien mit überschaubarem Aufwand zu prüfen, wurde ein spezieller Kurzzeit-Inhalationstest für Nanomaterialien entwickelt und erfolgreich eingesetzt [18]. Andere tierexperimentelle Studien haben entzündungsfördernde und fibrogene Wirkungen gezeigt, und auch kardiovaskuläre Effekte wurden beobachtet. Einige dieser Studien wurden allerdings mit unphysiologischen Expositionen wie z.B. durch intratracheale oder intraperitoneale Instillation durchgeführt. In Langzeitstudien konnte bei Ratten eine vermehrte Tumorentstehung in der Lunge gesehen werden; diese Befunde wurden als „Overload-Effekt“ aufgefasst, die auch mit größeren (nichtnano- 
skaligen) persistenten Partikeln beobachtet werden [19]. Neuere Untersuchungsergebnisse geben Anhaltspunkte für den Verdacht, dass sich nadelförmige Partikel aus Kohlenstoff, sogenannte „Multi-walled Carbon-Nanotubes“ (MWCT), zumindest nach intraperitonealer Injektion bei Mäusen wie Asbestfasern verhalten könnten [20]. Es gibt zwei Studien mit längerdauernder inhalativer Exposition von MWCNT. Beide zeigten bei Ratten dosisabhängige granulomatöse Veränderungen in den Lungen, wobei die Untersucher unterschiedliche No-Effect-Konzentration beobachteten [21,22]. Diese Unterschiede sind auf die Bewertung der Veränderungen im niedrigen Dosisbereich zurückzuführen.

Erfahrungen am Menschen liegen bisher nur in äußerst beschränktem Umfang vor. Einige spektakuläre Fallbeschreibungen, in denen zum Teil erhebliche Gesundheitsstörungen auf Benutzung „nanohaltiger“ Verbraucherprodukte oder aber Expositionen an Arbeitsplätzen zurückgeführt wurden, erwiesen sich bei näherer Analyse als nicht haltbar. Entweder enthielten die genannten Produkte gar keine Nanoobjekte oder, bei den Berichten von bestimmten chinesischen Arbeitsplätzen, waren die beschriebenen Expositionsbedingungen dort derart katastrophal, dass der Frage, ob hier Nanomaterial beteiligt war, keine weitergehende Bedeutung mehr beizumessen war [23].

In zwei Studien mit inhalativer Exposition gesunder freiwilliger Versuchspersonen gegenüber nanoskaligem Titandioxid konnten keine reproduzierbaren Kurzzeiteffekte auf Entzündungsmarker, verschiedene Parameter der Blutgerinnung und Herzfrequenzvariabilität gefunden werden [24,25]. Die Ergebnisse von Inhalationsstudien mit Dieselrußpartikeln, die Hinweise auf mögliche mutagene oder vaskuläre Effekte liefern, können nicht ohne Weiteres auf künstliche Nanomaterialien übertragen werden.

Epidemiologische Studien zum Zusammenhang zwischen partikulären Luftbelastungen und Mortalität oder Morbidität an Asthma- oder Herz-Kreislauf-Erkrankungen sind nicht hinreichend interpretierbar, da die Partikelbelastungen in der Regel nicht im Nanobereich gemessen wurden und darüber hinaus stark mit anderen Belastungsfaktoren wie z.B. Stickoxiden, Schwefeldioxid und sogar Verkehrslärm korreliert sind. Eine Übertragung dieser Studienergebnisse auf die Situation an Arbeitsplätzen ist auch deshalb nicht sinnvoll, weil die dort untersuchten Zielgruppen vorwiegend Kinder, Alte und chronisch Kranke waren, somit Personengruppen, die an Arbeitsplätzen nicht nennenswert vertreten sind.

Angesichts dieser lückenhaften Datenlage mag die über viele Jahrzehnte gewonnene arbeitsmedizinische Erfahrung mit Schweißern zunächst zur modellhaften Betrachtung herangezogen werden. Schweißrauche stellen ein komplexes Gemisch mit einem hohen Anteil von Nanomaterialien dar und repräsentieren in verschiedener Hinsicht ein „Worst-case-Szenario“: Die Partikelzahlen in der Luft beim Schweißen gehören zu den höchsten an Arbeitsplätzen gemessenen, die Partikel sind metallisch und damit von potenziell hoher intrinsischer Toxizität, sie sind teilweise ionisiert und damit hoch reaktiv oder katalytisch aktiv und sie enthalten Verunreinigungen mit kanzerogenen polyzyklischen aromatischen Kohlenwasserstoffen (PAHs). Zusätzlich ist eine Co-Exposition mit zahlreichen irritierenden oder toxischen Gasen wie z.B. Kohlenmonoxid, Stickoxiden und Ozon zu berücksichtigen $[26,27]$. Vor diesem Hintergrund ist der relative Mangel an belegten Gesundheitseffekten beruflicher Schweißrauchexposition bemerkenswert [28]. Eine Beeinträchtigung der Lungenfunktion wurde nur bei wenigen der am höchsten exponierten Personen beobachtet. Seltene Fälle von Lungenfibrosen wurden auf eine Mischexposition mit silikogenen Stäuben bei schlechten Belüftungsbedingungen zurückgeführt. Die BK 4115 - Lungenfibrose durch extreme und langjährige Einwirkung von Schweißrauchen und Schweißgasen (Siderofibrose) wurde im Juni 2009 in die Berufskrankheitenliste aufgenommen. Um einen Kausalzusammenhang zwischen dem Auftreten einer Lungenfibrose und einer beruflichen Exposition gegenüber Schweißrauchen/-gasen anzunehmen, wurde in der wissenschaftlichen Begründung zur BK 4115 die „bestimmte Personengruppe“ genauer abgegrenzt: „Versicherte nach einer mindestens etwa 10-jährigen beziehungsweise 15000-stündigen Schweißertätigkeit unter extremen Bedingungen, das heißt bei eingeschränkten Belüftungsverhältnissen, zum Beispiel in Kellern, Tunneln, Behältern, Tanks, Containern, engen Schiffsräumen etc.“

Wirkungen auf das Zentralnervensystem und die Reproduktionsfähigkeit wurden nicht oder uneinheitlich beschrieben oder auf andere Einwirkungen wie z.B. Strahlungswärme bezogen. Hinweise auf eine krebserzeugende Wirkung beim Menschen bestehen, können aber derzeit nicht als gesichert gelten; entsprechend werden Schweißrauche von der International Agency for Research on Cancer (IARC) auch nur als „möglicherweise krebserzeugend" eingestuft [29].

Dieser Vergleich kann selbstverständlich nicht dazu dienen, den Verdacht auf Gesundheitsgefahren durch synthetisches Nanomaterial zu entkräften. Er kann allerdings angestellt werden, um die Größenordnung dieser Gefährdung unter heutigen Arbeitsplatzbedingungen grob abzuschätzen.

\section{Vorsorgeuntersuchungen bei beruflicher Exposition \\ gegenüber Nanomaterialien}

Eine evidenzbasierte Grundlage für die Definition eines „nanospezifischen“ Untersuchungsprogramms liegt derzeit noch nicht vor $[4,30]$. Die im Zusammenhang mit Nanomaterialien diskutierten Wirkungen sind sowohl hinsichtlich ihrer Mechanismen als auch der potenziellen Zielorgane unspezifisch und können auch durch zahlreiche außerberufliche Faktoren bedingt werden. Entsprechende Befunde sind in der Allgemeinbevölkerung häufig anzutreffen. Sogenannte „innovative Marker“, wie Interleukine, Interferon, Gerinnungsparameter, NO-Exhalation, Herzfrequenzvariablilität u.v.m., sind als Untersuchungsinstrumente in der Praxis nicht sinnvoll einsetzbar, sondern können allenfalls in wissenschaftlichen Studien zur Anwendung kommen. Die etablierten und im medizinischen Alltag anwendbaren Verfahren mit bekannter Sensitivität und Spezifität für die Diagnostik solcher Erkrankungen, insbesondere bei symptomatischen Personen und in Risikopopulationen, können im Falle eines „positiven“ Befundes nicht zwischen beruflicher und nichtberuflicher Verursachung unterscheiden. Das hierdurch verursachte Dilemma ist an anderer Stelle ausführlich diskutiert worden [4].

\section{Risiko-Management beim Umgang mit Nanomaterialien $\nabla$}

Aus dem Umstand, dass das Risiko beim Umgang mit Nanomaterialien derzeit nicht ausreichend quantifizierbar ist, kann selbstverständlich nicht gefolgert werden, dass es vernachlässigbar sei. Da die Sekundärprävention durch Früherkennung in der momentanen Situation nicht gewährleistet ist, kommt der Primärprävention durch geeigneten Arbeitsschutz eine umso größere Bedeutung zu. Diese Prävention wird bevorzugt durch technische 
Maßnahmen der Expositionsvermeidung oder -minimierung erreicht, die bedarfsweise durch organisatorische und persönliche Schutzmaßnahmen ergänzt werden. Nach bisher vorliegenden Forschungsergebnissen kann hier das vorhandene Wissen um die Entstehung, räumliche Verteilung sowie Ausbreitungsvermeidung von Aerosolen einschließlich der Nutzung vorhandener Lüftungs- und Filtertechnik angewandt werden. Da bislang keine Expositionsgrenzwerte für Nanomaterial existieren, soll die Belastung am Arbeitsplatz die Hintergrundbelastung möglichst nicht übersteigen. Die Herstellung von und der Umgang mit Nanomaterialien im geschlossenen System wird unter Normalbedingungen als emissionsfrei eingeschätzt. Bei offener Handhabung kann durch geeignete Formulierung, beispielsweise als Dispersion oder Aufschlämmung, eine inhalative Belastung vermieden werden. Bei manchen Arbeitsschritten, wie z. B. Ein- oder Abfüllen, Mahlen, Konfektionieren oder bei Probenahmen, besteht unter Umständen trotz vorhandener Absaugeinrichtungen die Möglichkeit einer Staubemission. Hierbei, sowie bei einer Betriebsstörung, wie beispielsweise einer Leckage, sind persönliche Schutzmaßnahmen zu ergreifen. Atemschutzfilter der Klassen P2 und P3 bieten mit einer Durchlässigkeit von ca. 0,2\% bzw. 0,01\% einen ausreichenden Schutz vor inhalativer Belastung, wobei allerdings das Leckagevolumen bei nicht dicht schließendem Sitz der Maske zu berücksichtigen ist. Ein höheres Schutzniveau kann bei Bedarf durch Verwendung einer Vollmaske mit Partikelfilter der Klasse P3 oder durch ein umgebungsluftunabhängiges Atemschutzgerät erreicht werden. Eine dermale Exposition sollte entsprechend der üblichen Sicherheitsstandards beim Umgang mit Arbeitsstoffen vermieden werden. Hierzu werden geeignete Schutzhandschuhe und Einwegschutzoveralls verwendet, unter außergewöhnlichen Bedingungen auch Chemikalienvollschutzanzüge.

\section{Keine arbeitsmedizinische Vorsorge bei Umgang mit Nanomaterialien?}

In der Praxis erhalten Mitarbeiter an potenziell exponierten $\mathrm{Ar}-$ beitsplätzen häufig bereits arbeitsmedizinische Untersuchungsangebote aus anderen Anlässen, wobei Atemschutz und Wechselschicht die häufigsten Anlässe sein dürften. Die systematische Datenerhebung bei solchen Untersuchungsanlässen, zusammen mit einer möglichst genauen Dokumentation der jeweiligen Expositionsbedingungen im Sinne eines „Nanokatasters“, kann in der Zukunft wichtige Hinweise auf mögliche Gesundheitsrisiken geben, sei es durch die Erkennung von "sentinel health events“ oder durch die retrospektive epidemiologische Auswertung der so entstehenden Datenbasis.

\section{Danksagung \\ $\nabla$}

Die TEM-Aufnahmen wurden von Prof. Nancy Monteiro von der North Carolina State University zur Verfügung gestellt.

\section{Nanotechnology - New Challenges in Occupational Medicine \\ $\nabla$}

Nanomaterials are solid, particulate substances which are intentionally manufactured at the nano-scale and consist of nano-objects with at least one dimension between 1 and $100 \mathrm{~nm}$ (ICCA 2010). Naturally occurring and unintentionally produced substances at the nano-scale level are usually not considered as nanomaterial but as ultrafine materials (ultrafine particulate matter).

Nanomaterials may possess different chemical or physical properties compared to those found in the respective bulk materials. This is indeed why they are produced and used in nanotechnology. However, apart from these intended new properties nanomaterials may also exert toxic effects that might not be simply derived from existing knowledge of the effects of the bulk material. Dermal Penetration studies suggest that many Nanomaterials are not absorbed through the skin. Inhalation studies suggest that nanomaterials may cause unspecific inflammatory and fibrogenic reactions in the lung, similar to those known from exposures to coarse particles. Nanoparticles, however, might elicit stronger effects and could affect additional organs in the body.

Exposure assessment of nanomaterials is currently not sufficiently developed and harmonized. Exposure measurement techniques for nanomaterials by mass, surface, chemical species or number of particles still need to be standardized.

Up to now, human data on specific health effects of nanomaterials do not exist. Therefore, occupational medical surveillance targeted to specific health effects is currently not possible. Against this background risk assessment and exposure control in workplaces is of paramount significance.

\section{Literatur}

1 Oberdörster G, Oberdörster E, Oberdörster J. Nanotoxicology - an emerging discipline involving studies of ultrafine particles. Environ Health Perspect 2005; 113: $823-839$

2 Salomon M. Gesundheitsrisiken durch synthetische Nanomaterialien. Umweltmed Forsch Prax 2009; 14: 7-22

3 Bundesministerium für Bildung und Forschung (BMBF). Nanotechnologie - eine Zukunftstechnologie mit Visionen. Im Internet: www.bmbf. de/de/nanotechnologie.php; Stand: 2.1.2010

4 Nasterlack M, Zober A, Oberlinner C. Considerations on occupational medical surveillance in employees handling nanoparticles. Int Arch Occup Environ Health 2008; 81: 721 - 726

5 Wittmaack $K$. In search of the most relevant parameter for quantifying lung inflammatory response to nanoparticle exposure: particle number, surface area, or what? Environ Health Perspect 2007; 115: $187-$ 194

6 Phillips JI, Green FY, Davies JCA, Murray J. Pulmonary and Systemic Toxicity Following Exposure to Nickel Nanoparticles. American Journal Of Industrial Medicine 2010; 53: $763-767$

7 Landsiedel R, Ma-Hock L, Kroll A et al. Testing metal-oxide nanomaterials for human safety. Advanced Materials 2010; 22: 2601-2627

8 Baroli B, Ennas MG, Loffredo $F$ et al. Penetration of metallic nanoparticles in human full-thickness skin. J Invest Dermatol 2007; 127: $1701-1712$

9 Ryman-Rasmussen JP, Riviere JE, Monteiro-Riviere NA. Penetration of intact skin by quantum dots with diverse physicochemical properties. Toxicol Sci 2006; 91: 159-165

10 Nohynek G, Lademann J, Ribaud C et al. Grey goo on the skin? Nanotechnology, cosmetic and sunscreen safety. Crit Rev Toxicol 2007; 37: $251-277$

11 Lademann J, Weigmann H-J, Rickmeyer C et al. Penetration of titdnium dioxide microparticles in a sunscreen formulation into the horny layer 
and the follicular orifice. Skin Pharmacol Appl Skin Physiol 1999; 12 : $247-256$

12 Gamer AO, Leibold E, van Ravenzwaay B. In vitro absorption of microfine zinc oxide and titaniumdioxide through porcine skin. Toxicol ln Vitro 2006; 20: $301-307$

13 Cross SE, Innes B, Roberts MS et al. Human skin penetration of sunscreen nanoparticles: In vitro assessment of a novel micronized zinc oxide formulation. Skin Pharmacal Physiol 2007; 20: 148-154

14 Inman $A O$, Landsiedel $R$, Wiench $K$ et al. Assessment of UVB-damaged skin in vivo with sunscreen formulations containing titanium and zinc nanoparticles. 49th Annual Meeting of the Society of Toxicology, Salt Lake City, UT, USA: 2010

15 Monteiro-Riviere NA, Wiench K, Landsiedel R et al. In vitro penetration studies of four sunscreen formulations containing titanium and zinc nanoparticles in UVB-damaged skin. 49th Annual Meeting of the Society of Toxicology, Salt Lake City, UT, USA: 2010

16 Zhang LW, Monteiro-Riviere NA. Assessment of quantum dot penetration into intact, tape stripped, abraded, and flexed rat skin. Skin Pharmacal Physiol 2008; 21: $166-180$

17 Wörle-Knirsch JM, Pulskamp K, Krug HF. Oops they did it again! Carbon nanotubes hoax scientists in viability assays. Nano Lett 2006; 6: 1261 1268

18 Ma-Hock L, Burkhardt S, Strauss V et al. Development of a short-term inhalation test in rats using nano-titanium dioxide as a model substance. Inhalation Toxicology 2009; 21: $102-118$

19 Borm PJA, Robbins D, Haubold $S$ et al. The potential risks of nanomaterials: a review carried out for ECETOC. Part Fibre Toxicol 2006; 14: 11

20 Poland CA, Duffin R, Kinloch I et al. Carbon nanotubes introduced into the abdominal cavity of mice show asbestos-like pathogenicity in a pilot study. Nature nanotechnology 2008; 3: 423-428
21 Pauluhn J. Multi-walled carbon nanotubes (Babytubes): Approach for derivation of occupational exposure limit. Reg Pharm Toxicol 2010; 57: $78-89$

22 Ma-Hock L, Treumann S, Strauss V et al. Inhalation toxicity of multiwall carbon nanotubes in rats exposed for 3 months. Toxicol Sci 2010; 112: $468-481$

23 Song Y, $\mathrm{Li} X, \mathrm{Du} \mathrm{X}$. Exposure to nanoparticles is related to pleural effusion, pulmonary fibrosis and granuloma. Eur Respir J 2009; 34: 559 567

24 Beckett WS, Chalupa DF, Pauly-Brown A et al. Comparing inhaled ultraWne versus Wne zinc oxide particles in healthy adults: a human inhalation study. Am J Respir Crit Care Med 2005; 171: 1129-1135

25 Scharrer E, Hessel H, Kronseder A et al. Heart rate variability, hemostatic and acute inXammatory blood parameters in healthy adults after short-term exposure to welding fume. Int Arch Occup Environ Health 2007; 80: 265-272

26 Zober A. Symptoms and findings at the bronchopulmonary system of electric arc welders. Zbl Bakt Hyg 1981; 173: 92-119

27 Wieners D, Latza U, Baur X. Inhalative Belastungen durch Schweißverfahren. Zbl Arbeitsmed 2000; 50: 38-44

28 Antonini JM. Health effects of welding. Crit Rev Toxicol 2003; 33: 61 103

29 IARC. Chromium, nickel and welding. In: WHO, ed. IARC monographs on the evaluation of carcinogenic risks to humans. vol. 49. Geneva: IARC Monogr Eval Carcinog Risks Hum; 1990: 1 - 648

30 Trout $D$, Schulte PA. Medical surveillance, exposure registries, and epidemiologic research for workers exposed to nanomaterials. Toxicology 2010; 269: $128-135$ 